UCRL- 92806

PRE:DRINT

\title{
SEISMIC VERIFICATION OF UNDERGROUND EXPLOSIONS
}

\author{
Lewis A. Glenn
}

This paper was prepared for submittal to

Fourth Topical Conference on Shock Waves

in Condensed Matter,

Spokane, Washington

July 21-25, 1985

June 1985

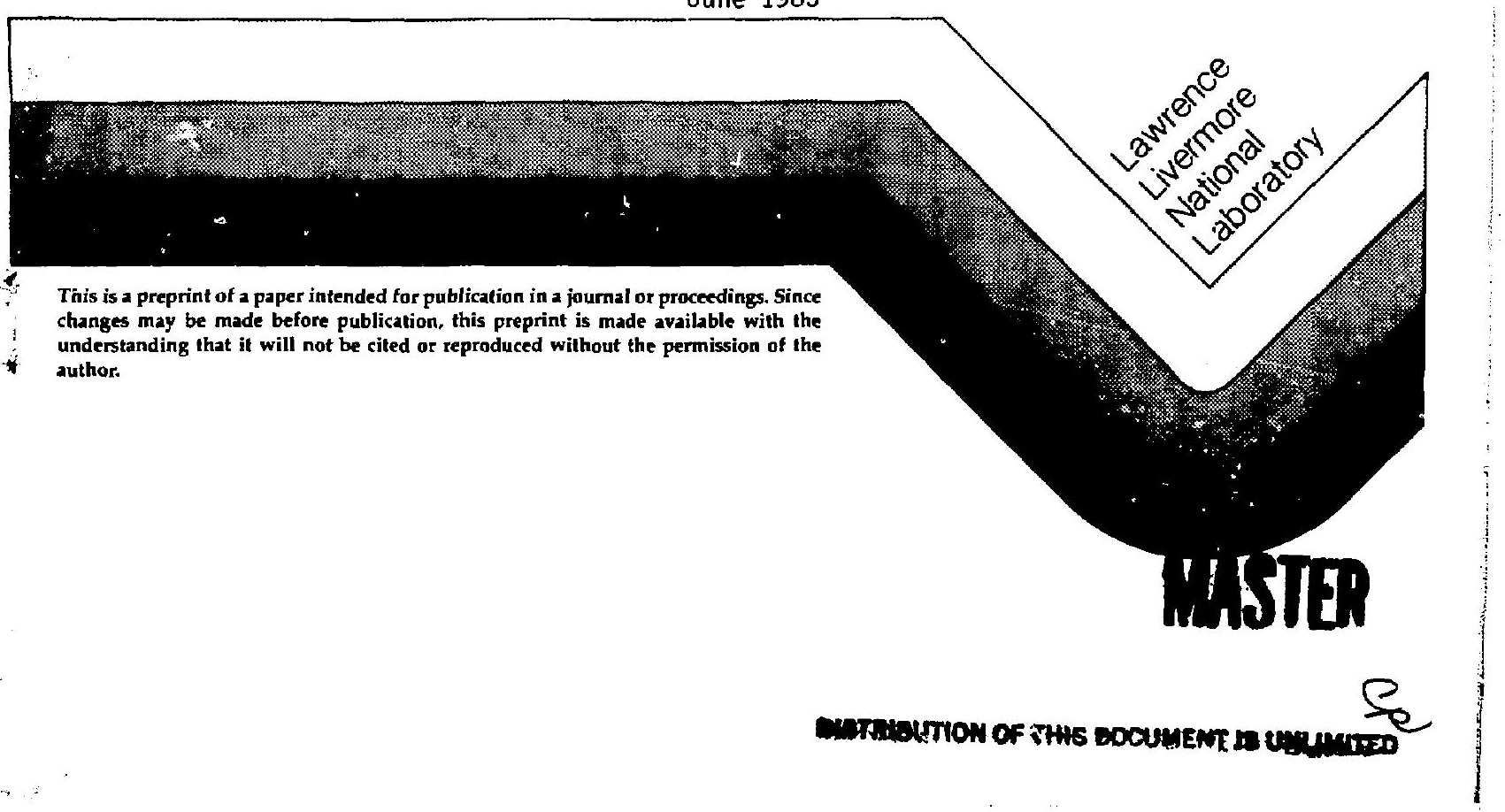


UCRL--92806

DE85 013979

SEISHIC VERIFICATION OF UNDERGROUND EXPLOSIONS *

Lewis A. Glenn

Lawrence Livermore National Laboratory

Physics Departwent

Livermore, CA 94550

\section{INTRODUCTION}

Bans or limitations on nuclear weapons tests are one of the many types of arms control measures that have been attenpted over the years. The first nuclear test agreement, the test moratorium, was made in 1958 and lasted until the Soviet Union unilaterzlly resuned testing is the atmosphere in 1961. It was followed by the Limited Test Ban Trea-y of 1963, which prohibited nuclear tests in the atwosphere, in outer spece, and underwater. In 1974 the Threshold Test Ban Treaty (THBT) was signer. This treaty limited underground tests after warch 1976 to a mimu yield of 250 kilotons (kt). The TTBT was followed by a treaty limiting peaceful nuclear explosions and, although neither of these was ever ratified by the U.S. Senate, both the United States and the Soviet Union claim to be abiding by the 150-kt yield linit. A comprehensive test ban treaty (CTBT), prohibiting all testing of nuclear weapons, has also been discussed and there is widespread public interest in this idea. However, a verifiable CTBT is a contradiction in terms. No monitoring technology can offer absolute assurance that very-low-yield illicit explosions have not occurred.

\section{VERIFICATION RROCESS}

The principal tools for monitoring compliance with a CTBT are seigaic networks and surveillance satellites. On-site inspections wight also be required to resolve ambiguous events. Satellites are actually of limited use since it is possible to carry out low-yield explosions in buried cavities without resulting in any visible ground-surface notion. Moreover, test activities, including the required excavations, can be associated with mining or other large-scale industrial undertakngs. The critical element of the wonitoring system is thus the network of seismic stations, and in particular the in-country stations.

Internal stations provide wuch wore useful data than do stations situated outside the borders of testing nations. The external stations are generally so far from potential evasion sites that they record the motion of only a few waves from the larger events (those with seisuic magnitudes, mb. greater than about 4 on the Richter scale) and high *Work performed under auspices of USDOE by LLNL under \#W-7405-Eng-48. 
fzequencies have been significantly reduced by attenuation and scattering throughout the earth. The larger amplitudes recorded by the internal stations, for the same event, result in an increase in detection capability. The multiple waves and higher frequencies improve the ability to distinguish between explosions and earthquakes. These two functions, detection and identification, are the main elements in the verification process.

The detection capability of a seismic network is specified by the seismic magnitude of the smallest source that can be detected within its assigned territory, with a specified degree of confidence. Theoretical estimates of detection capabilities as low as $m_{b}=2.7$ have been made fol the Soviet Union, at the 908 confidence level, assuming a very extensive network of internal stations ${ }^{1}$ even lower estimates result if lower confidence limits are acceptable. 2

Given that waves from an event have been detected, the source must be identified as a nuclear explosion, a chemical explosion, or an earthquake. Many discriminants have been proposed. First, the located depth of the source is useful since current drilling limits are less than about $12 \mathrm{~km}$. Therefore, explosions are not likely to be responsible for signals originating at greater depths.

Second, the areas in which large magnituce earthquakes occur are fairly well known. A strong seismic signal originating at a shallow depth in say Ransas is much more likely to be the result of an explosion than an earthquake; the same would not necessarily be true for California. However, areas that are seismically inactive at high magnitude levels may not be so at the low levels demanded for CTB verification. For example, a recent study of parts of Louisiana, Cklahoma, and Texas which had shown only one earthquake of magnitude 4.5 or greater in 8 years, revealed that almost one earthquake per day occurred in the magnitude range 2.0 to 3.9.3 Similar results can be expected in many granitic regions so that the areal discriminant is probably of limited use for small events.

For those large events that are not eliminated by depth or location, one of the most useful discriminants is based on the ratio of surface-wave to body-wave magnitudes ( $M_{S}: m_{b}$ ). If an explosion and an earthquake have the same body-wave magnitude, the surface-wave magnitude for the earthquake is generally larger.4,5 It has yet to be proven that $M_{s}: m_{b}$ is useful at low magnitudes, especially when explosions are set off in long tunnels or odd-shaped cavities (see below).

A number of other promising regional discriminants have been suggested, based on differences in the spectral content and radiation pattern of the recorded signal. 6

\section{EVASION OPPORTUNITIES}

Detection of an explosion can be avoided by insuring that the signal generated is below the background noise level, by emitting "norinal" signals at times of high noise, or both. If the noise only partially masks the signal, detection reay occur but identification may be impaired. Reducing the signal strength at the receiving station may be accomplished either by reducing the coupling of the explosive energy into the earth at the source or by choosing (through site selection) a path from the source to the seismic station through a region that absorbs significant seisnic energy. 
To understand the significance of decoupling it is necessary to interpret the detection capability of a network in terms of explosive yield, as illustrated in Figure 1. The geisnic body-wave agnitude is plotted against the yield in kilotons. The data are representative of U.S. experience at the levada Test site (NTS). 7 Various investigators have presented evidence that a given explosion in the soviet Onion registers several tenths of a nagnitude unit higher than the same explosion at the NTS 8,9 (this in fact was a principal factor in the recent controversy over alleged violations of the Threshold Test Ban Treaty).

The upper band in Figure 1 shows the seisnic magnitude that would be generated by explosions that are tamped, or well-coupled to hard rock. The middle line drawn in this band is based on the "granite" representation In ref. 7 ; the lower (ref. 10) and higher (ref. 1) lines derive from independent analyses made for hard rock. The widening spread at low yield is due to the facts that there are very few hard-rock data below $1 \mathrm{kt}$, that these are characterized by considerable variability, and that there are probleas in obtaining a consistent definition of magnitude as well. Under these conditions, if the lower linit of detectability corresponds to $m_{b} \approx 2.7$, the maximum explosive yield would be approximately $0.1 \mathrm{kt}$.

The midale band in Figure 1 shows the effect of detonating the explosion in the deep, dry alluvial deposits at the NHS. For the sane body-magnitude, the explosive yield would be about 10 times greater in alluvium than in hard rock. The reason is that the alluviun is porous and a large fraction of the source-energy is dissipated by pore collapse.

The lower band in Figure 1 corresponds to explosions in large cavities and represents by far the most important potential for evasion.

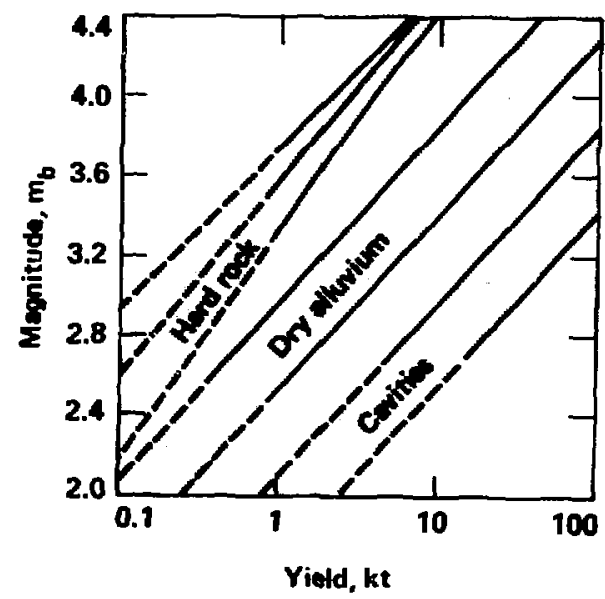

Fig. 1. Soismic body-wove emplitude vs. explosive yield for different explosion environments. The relationships ere besed on U.S. date from the Novada Teat Sito. 


\section{CAVITY DECOUPLING}

The idea of using a large cavity to muffle the seismic signal from an underground explosion was first proposed 25 years ago by Albert Iatter and Hans Bethe at an early conference on the discontinuance of nuclear tests. 11 They calculated that the strength of the distant seismic signal in an elastic medium is determined prinarily by the volume of the cavity created by the explosion. If the explosion is set off in a large cavity, then it is the differential increase that determines the signal anplitude. Woreover, if the cavity is just large enough so that its walls suffer only elastic deformation, the distant signal, for a given yield, is minimized and it does not pay to make the cavity any bigger. Inelastic behavior causes increased coupling because the medium is unable to support shear and can thereby flow and thus undergo large displacements.

Experimental confirmation of the cavity-decoupling theory was first obtained in a series of experiments collectively called CONBOY, that was conducted with chemical explosives in a Louisiana salt mine in 1960.12 salt was chosen as the test medium because of its homogeneity in situ and because of the ease in which a cavity can be constructed and maintained. The ratio of the seismic amplitude in the tamped explosion to that obtained when the same yield is generated in a excavated cavity is called the decoupling factor; a decoupling factor of 100 was measured in COFBOY.

Confirmation of cavity decoupling in a nuclear experiment was made in the STERLING event $(0.38-k t$ yield $) 13$, which was fired in the Tatum salt dome (near Hattiesburg, Mississippi) on December 3, 1966, more than two years after the SALMON event $(5.3-\mathrm{kt}$ yield) 14 created the cavity. A maximum decoupling factor $70 \pm 20$ was determined for STERLING, indicating that full decoupling may not have been achieved. It was known prior to STERLING that the SALMON cavity (17.4-m radius) was too small to fully decouple the explosion; this would have required roughly 608 more volume. Horeover, although creating the cavity with an earlier explosion was cheaper than conventional mining techniques, the salt structure around the cavity was thereby weakened (the theory predicts the decoupling factor to be proportional to the rigidity modulus of the surrounding medium).

The significance of these results can be seen when they are rombined with the lower limit of seismic detectability. As illustrated in Figuxe 1, an $m_{b}$ measurement of 2.7 now corresponds to a 5-kt explosion (instead of the taraped, hard-rock value of $0.1 \mathrm{kt}$ ), even if the decoupling factor is only 50. Decoupling factors several times higher are predicted if the cavity can be excavated in a stiffer medium, such as granite. 15

Notwithstanding the experimental evidence, recent studies have questioned the utility of cavity decoupling.16,17 Figure 2 illustrates the problem. The spectral variation of the seismic amplitude is plotted, for a fixed yield, for both tamped and cavity-decoupled explosions. In either case it is observed that only modest changes occur as the angular frequency is increased to the neighborhood of the eigenfrequency, $\omega \approx c / R$, where $c$ is the wave speed and $R$ the elastic radius, after which severe damping occurs. For the carity-decoupled explosion, this "corner" frequency is based on the cavity radius, $\mathrm{R}_{\mathrm{D}}$. For the tamped explosion, the radius at which the shock wave attenuates to an elastic wave, $R_{T}$, is much larger. A heuristic explanation for this derives from the fact that the radial monentum increase in a strong explosion is proportional to the square-root of the mass engulfed by the blast wave, which is evidently

$$
-4-
$$




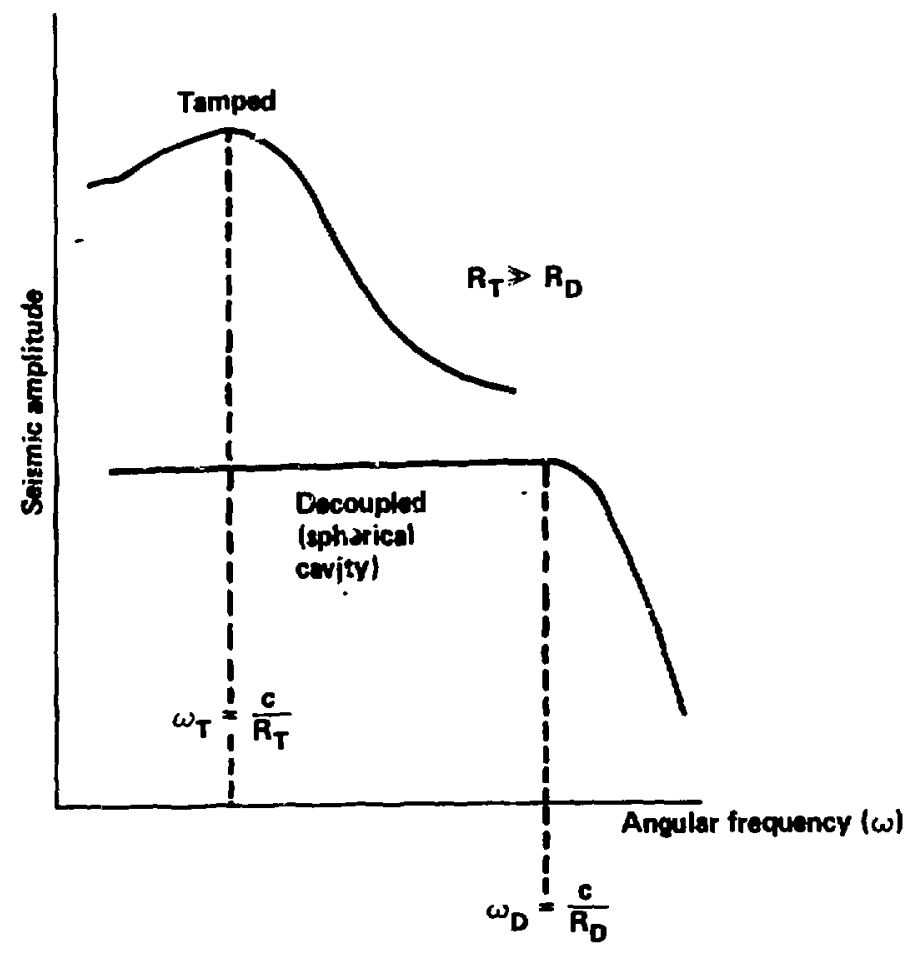

Fig. 2. Spectral variation of seismic amplitude for tamped and carity-decoupled explosions. Both abscissa and ordinate scales we logarithmic. The ratio of the tamped to the decoupled amplitude is called the decoupling factor and theoretically decreases at frequencies above $\omega_{T}$.

many times larger when the cavity is initially filled with rock than with air. Thus, even though the amplitude of the tamped explosion is 50 or more times higher for $\omega<w_{\mathrm{T}}=c / \mathrm{R}_{\mathrm{T}}$, it may be much less in the region $\omega_{\mathrm{T}}<\omega<\omega_{\mathrm{D}}=c / R_{\mathrm{D}}$. The decoupling factor can therefore be expected to decrease substantially in this frequency interval. The effect is of no signficance for teleseismic monitoring, since frequencies greater than $w_{T}$ are effectively attenuated by the earth. There is some evidence, however, that efficient high-frequency transmission can occur at regional distances in certain geographical regions of importance. Mlso, earth noise decreases with increasing frequency, aiding in the signal analysis.

One remedy would be to increase the size of the decoupled cavity. However, co fully decouple a 5-kt explosion requires a salt cavity with a radius of $4 \mathrm{~g} \mathrm{~m}^{18}$, so that it is infeasible, or at least inpractical, to excavate a sphere of the required size. An alternate wethod of increasing the characteristic size of the cavity is to connect long tunnels to the explosion chamber. These could easily extend beyond distanceg corresponding to $R_{T}$. Recent calculations have shown that, by exploiting c. structive interference effects, elongated cavities may serve as effective low-pass $\mathrm{filters}$ for seismic radiation in directions roughly 
parallel to the long axis.19,20 For cylindrical tunnels, for example, the corner frequence varies inversely with length or, relative to a sphere with the same volume, with the 2/3-power of the aspect ratio.

Construction of such facilities would be easier and cheaper than spherical cavities of the same volume. Hard-rock excavations of the required size would nevertheless be snormous and costly undertakings, difficult to conceal. Because such formations are often located in regions of very active seismicity, however, cavities excavated there would seriously complicate the already difficult problem of discriminating between low-yield explosions and enrthquakes.

The excavation of large underground cavities may not be hard to disguise from satellite obsurvation if the activity is associated with mining or other large-scale industrial undertakings. In salt, the least expensive method is solution mining. A 1970 study indicated that a $50-\mathrm{m}$ radius cavity could be solution-mined in the Tatum salt dome at a cost under \$loM. 21 By 1977 the cost was put at $\$ 20 \mathrm{M}$, but it was determined that a single cavity might be useful for up to 25 tests, performed over a period of two years. 22 Reuse of a cavity would probably be an important consideration since realistic present-day costs are undoubtedly still higher. With salt, however, there is always the SALMON option, i.e., to create a primary cavity with a tamped explosion, and then to enlarge it in steps by exploding in it larger and larger devices. It is possible that this technique may already have been employed by the Soviets in their Peaceful Nuclear Explosion (PNE) program, in which numerous nuclear explosions have been conducted for the announced purpose of storing the natural gas condensates from the Orenburg and Astrakhan fields. 23

\section{REPERENCES}

1. H. J. Hannon, Jr., Science, 227, 251-257 (1985).

2 L. R. Sykes and J. F. Evernden, Scientific American, 247, 47-55 (1982) .

3. D. Racine and P. Kiouda, "Seismicity of the Salt Areas of Texas, Louisiana, Oklahoma, and Ransas," Teledyne Geotech Rept. AL-79-3 (1979).

4. O. Dahlman and H. Israelson, Monitoring Underground Nuclear Explosions (Elsevier Scientific Publishing, New York, 1977) .

5. C. E. Husebye and S. Mykkelveit, "Identification of Seismic Sources - Earthquake or Underground Explosion, NATO Advanced Study Institute Series," 74, Series C (D. Reidel Publishing Co.., Boston, 1981).

6. P. Pomeroy, W. Best, and T. McEvilly, T. Bull. Seism. Soc. Amer., 72 (6B), S89-S130 (1982).

7. B. A. Bolt, Nuclear Explosions and Earthguakes (H. H. Freeman and Co., San Francisco, 1976, p. 167).

8. J. F. Evernden, Bull. Seism. Soc. Amer., 66, 549-592 (1976).

9. P. Marshall, D. Springer and H. Rodean, Eeophys. J., 57, 609-638 (1979).

10. J. F. Evernden, J. Geophys. Res., 75, 1028-1032 (1970) .

11. A. L. Latter and $H$. Bethe, in Verbatim Record of the 7 th Meeting of Technical Horking Group 2 , Conference of the Discontinuance of Nuclear Weapons Tests, held in the Palais des Nations, Geneva, Switzerland, December 2, 1959 GEN/DNT/TwG. .:/PV.7. January 15 (1960). 
12. R. F. Herbst, G. C. Werth, and D. :. Springer; J. Geophys. Res., 66 959-978 (1961).

13. D. Springer, M. Denny, J. Healy, and W. J. Mickey, J. Geophyg, Res,, 73, 5995-6011 (1968).

14. D. Rawson, R. Taylor, and D. Springer, Maturwissenschaften, 54, 525-531 (1967).

15. A. L. Latter, R. E. LeLevier, E. A. Martinelli, and w. G. J. MacMillan, J. Geophys. Res., 66, 943-946 (1961).

16. R. R. Blandford, Bull. Seism. Soc. Amer., 72, S60-S87 (1982).

17. D. R. Larson, "Spherical Wave Propagation in Elastic Media and its Application to Energy Coupling for Tanped and Decoupled Explosions," Lawrence Livermore National Laboratory Rept. UCRL-52655 (1979).

18. L. A. Glenn, "Cavity Decoupling and the Evasion of a Comprehensive Test Ban," Lawrence Livermore National Laboratory Rept. UCRL-90321 (1984).

19. I. A. Glenn, A. J. C. Ladd, B. Moran, and R. A. Wilson, Geophys. J. R. astr. Soc., 81, 231-242 (1985).

20. L. A. Glenn, B. Moran, A. J. C. Ladd, K. A. Hilson and J. A. Rial, submitted for publication in Geophys. J. R. astr. Soc., Lawrence Livermore National Laboratory Rept. UCRL-92337 (1985).

21. Project Payette Final Sumary Report, prepared by Fenix Scisson, Inc., Tulsa, Oklahoma, March (1970).

22. L.A. Grove, H. W. Hubbard, and E. A. Martinelli, "Feasibility of a Decoupled Test Facility in a Salt Dome," R\&D Associates, P.O. Box 9695, Marina del Rey, CA 90291, Rept. RDA-TR-104900-013, April (1977).

23. I. Y. Borg, Energy and Technology Beview; Lawrence Livermore National Laboratory, 36-37, May (1983). 